\title{
Thrombolytics, still the first therapeutic weapon against acute ST-segment elevation myocardial infarction
}

\author{
Fernando L Benaim"**, Sebastian R Fonseca", Hernan C Arazi, Mailen Blanco and Raul Ferreyra \\ Department of Cardiology Division, Sanatorio de la Trinidad Mitre, Sanatorio de la Trinidad Palermo, Buenos Aires, Argentina \\ \#Equally Contributed
}

\begin{abstract}
Cardiovascular disease (CVD) is the leading cause of death worldwide, being coronary events the most common cause of CVD. In the case of an acute STsegment elevation myocardial infarction, the conventional treatment involves the reperfusion of the culprit artery as soon as possible as angioplasty has replaced the thrombolysis therapy.

We present two patients with acute ST-segment elevation myocardial infarction. The first patient showed an ST-segment elevation of the anterolateral wall, and the second one, in the inferolateral wall. In both cases we performed rapid reperfusion with thrombolytics (ischemic time less than 30 minutes), with good response.

The first patient showed a lesion in the subendocardial myocardium in the late gadolinium enhancement in cardiovascular magnetic resonance imaging.

Taking into account the time delays of patients-derivation and door-to-balloon time in Argentina, these cases reinforce the indication of thrombolytics as the treatment of choice.
\end{abstract}

\section{Introduction}

Cardiovascular disease is still the leading cause of death worldwide. Coronary artery disease represents $30 \%$ of all cardiac deaths. The estimated average incidence of acute ST-segment elevation myocardial infarction (STEMI) is between 50-60 patients/100.000 inhabitants according to European and American data [1]. Reperfusion therapy is key for this condition. It is widely known that sooner the blood flow is restored, better will be the recovery of the global function and lower the mortality rate. The advent of cardiac catheterization has displaced the thrombolytic therapy. However, when both strategies are compared, but with an early regime (less than 120 minutes) of the first against the second one, it has been observed that all-cause mortality at 5 years was lower in patients diagnosed with acute STEMI, with statistical significance, in those receiving reperfusion with rTPA [2].

Taking into account the difficulties regarding the satisfaction of the appropriate time of reperfusion in our country, the delay of patients to seek medical care and the delays of hemodynamic services, or, even worst, the delays in referral to centers with reperfusion capabilities, we present two cases of acute STEMI receiving an ultraearly thrombolytic therapy.

\section{Case 1}

72-year-old sedentary woman, ex-smoker, with a history of hypertension and ischemic stroke with no sequelae 20 years ago. She had chronic obstructive pulmonary disease without oxygen therapy.

The patient was accompanying a relative in our clinic when she presented a feeling of imminent death associated with dyspnea in functional class IV. She was taken to the Emergency Department.

Physical examination revealed hemodynamic instability, sweating, paleness and coldness of the lower limbs. As the patient presented severe respiratory insufficiency, she received sedation to facilitate endotracheal intubation and mechanical ventilation.

Following sedation, the patient suffered a cardiac arrest that required two cycles of cardiopulmonary resuscitation and one vial of adrenaline was administered; heart rate normalized afterwards.

Patient electrocardiogram at admission revealed sinus tachycardia (130 beats/minute) and convex elevation in anterolateral wall indicative of acute myocardial infarction (Figure 1). We called the Hemodynamics Service, who estimated a delay of at least 120 minutes. The patient was then administered a loading dose of aspirin and clopidogrel, anticoagulation with heparin sodium and a gastric protection agent. Due to the patient's hemodynamic instability, she was administered an alteplase infusion, with a total ischemic time of 20 minutes and a door-to-needle time of 15 minutes. A cinecoronography was performed at 110 minutes. It revealed a unique mild-to-moderate lesion in the proximal segment of the anterior descending artery. The patient left the hemodynamic room with a Swan-Ganz catheter that showed a pattern consistent with cardiogenic shock; inotropes (dobutamine) were started.

${ }^{\star}$ Correspondence to: Fernando Leonel Benaim, Department of Cardiology Division, Sanatorio de la Trinidad Mitre, Sanatorio de la Trinidad Palermo, Buenos Aires, Argentina, E-mail: leonelbenaim@gmail.com

Key words: acute myocardial infarction, culprit artery, thrombolytics, early reperfusion strategy, cardiac magnetic resonance

Received: March 02, 2020; Accepted: March 10, 2020; Published: March 19, 2020 
Positive reperfusion criteria were observed: reduction of STsegment elevation and elevation of high-sensitivity troponin by 10 . Due to sedation, precordial pain could not be assessed.

Within the acute context of the condition, a Doppler echocardiography was performed. This test showed akinesia of anterior and apical wall, with severe left ventricular enlargement and severe dysfunction, without affecting the right ventricle (Figure 2).

A new Doppler echocardiography was performed 48 hours later. It revealed preserved dimensions and thickness. Preserved left ventricular systolic function. Apical hypokinesia. Moderate pericardial effusion, without involvement of right cavities.

The patient progressed satisfactorily. Inotropes were suspended at 72 hours and she was extubated at 48 hours.

She started receiving beta blockers, vasodilators and dual antiplatelet therapy.

30 days after discharge, on an outpatient basis, she underwent a cardiac magnetic resonance that demonstrated good left ventricular systolic function with moderate pericardial effusion and late gadolinium enhancement at intramyocardial mid-lateral ventricular level with subendocardial involvement that suggests being coronary related (Figure 3).

Patient is asymptomatic and presented no complication until now.

\section{Case 2}

This case involves a 61-year-old male patient with hypertension, dyslipidemia, overweight (BMI $\left.28.4 \mathrm{~kg} / \mathrm{m}^{2}\right)$, with a history of inferior

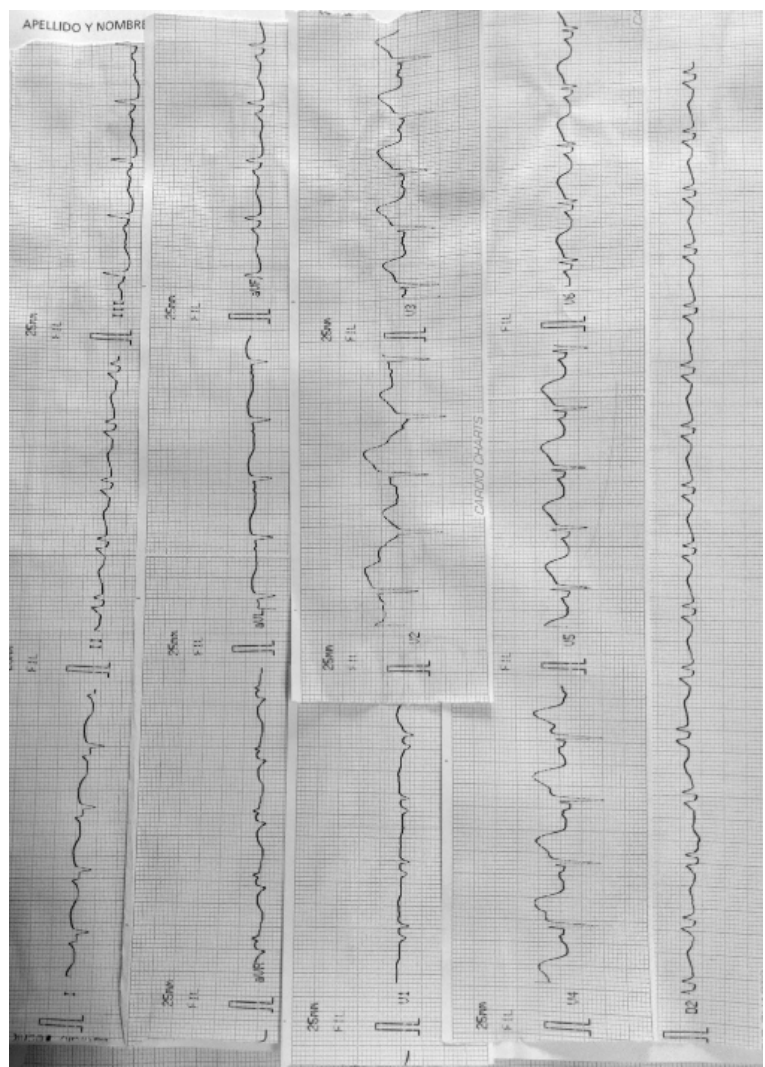

Figure 1. Convex elevation in anterolateral wall indicative of acute myocardial infarction

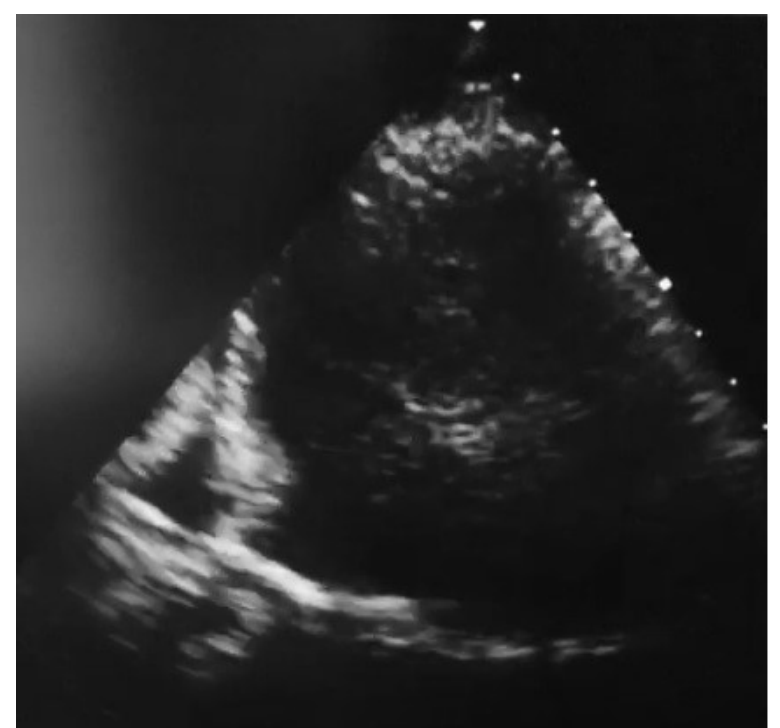

Figure 2. Akinesia of anterior and apical wall, with severe left ventricular enlargement and severe dysfunction, without affecting the right ventricle

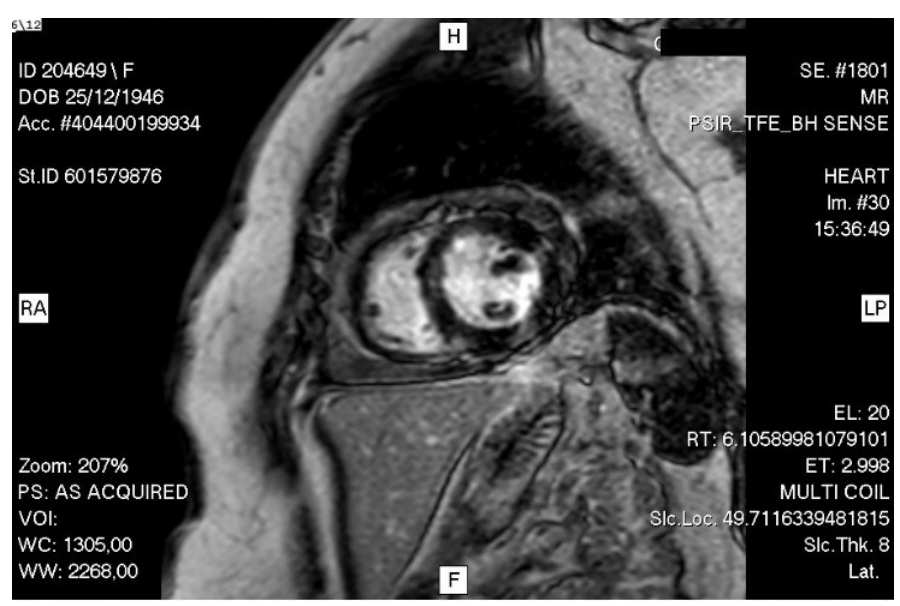

Figure 3. Good left ventricular systolic function with moderate pericardial effusion and late gadolinium enhancement at intramyocardial mid-lateral ventricular level with subendocardial involvement that suggests being coronary related

acute myocardial infarction (AMI), with Killip class I. The patient required the colocation of a conventional stent (BMS) in the right coronary artery (RCA) in 2012.

In 2015, a cinecoronography (CCG) was performed on an outpatient basis. The study revealed restenosis intra-stent of the RCA, plus severe injury in mid and distal segment; moderate lesion of anterior descending artery (DA) in mid segment; severe injury of second obtuse marginal branch of circumflex artery (CA). Implantation of one DES stent to proximal restenosis of DA and two BMS stents for injuries in mid and distal segment was required.

The patient comes to the emergency department, complaining of precordial pain. He is diagnosed with AMI without ST elevation, showing positive cardiac biomarkers (troponin 119.6 and $187 \mathrm{pg} /$ $\mathrm{mL}$ ). Anticoagulation with heparin sodium and IV vasodilators were started. The patient showed clinical and electrical improvement. A few hours later he progressed to AMI with inferior and lateral ST-segment elevation (Figure 4), reason why he was administered dual antiplatelet therapy combining aspirin and ticagrelor. Thrombolytics (rTPA) were used, achieving an ischemic time of 25 minutes, with positive 
reperfusion criteria (ST reduction of more than 50\% (Figure 5), pain disappearance and enzyme elevation, troponin $400 \mathrm{pg} / \mathrm{mL}$ ).

The patient then presented a third-degree AV block, with a pause greater than 5 seconds (evidenced by telemetry), reason why a CCG was performed urgently. The CCG showed the left main coronary artery without relevant angiographic injuries; DA with mild injury in mid segment; CA with serious injury in lateral-ventricular (LV) branch; dominant RCA, presents stents in proximal, mid and distal segment with occlusive restenosis in its mid segment. Patient received heterocollateral circulation (Figure 6).

A balloon coronary angioplasty (PTCA) was performed in chronic occlusion of proximal RCA but did not result. Then it was decided to perform a PTCA with implantation of 1 DES stent in severe lesion of LV branch of CX. This lesion was understood as culprit vessel, in the context of a patient with RCA chronic occlusion.

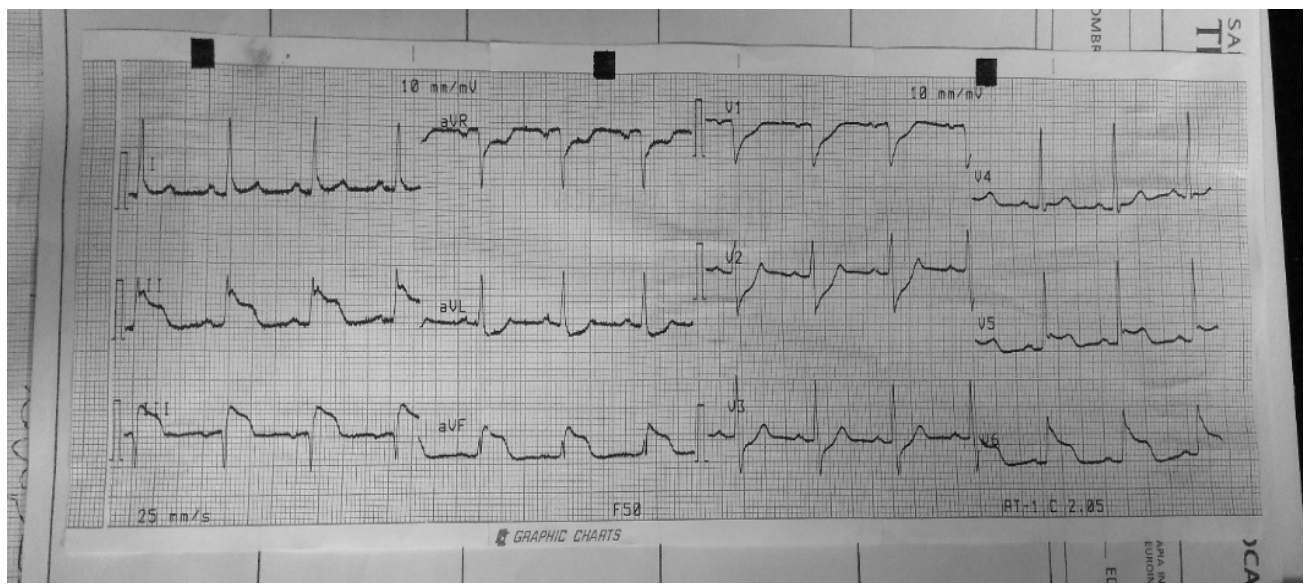

Figure 4. AMI with inferior and lateral ST-segment elevation

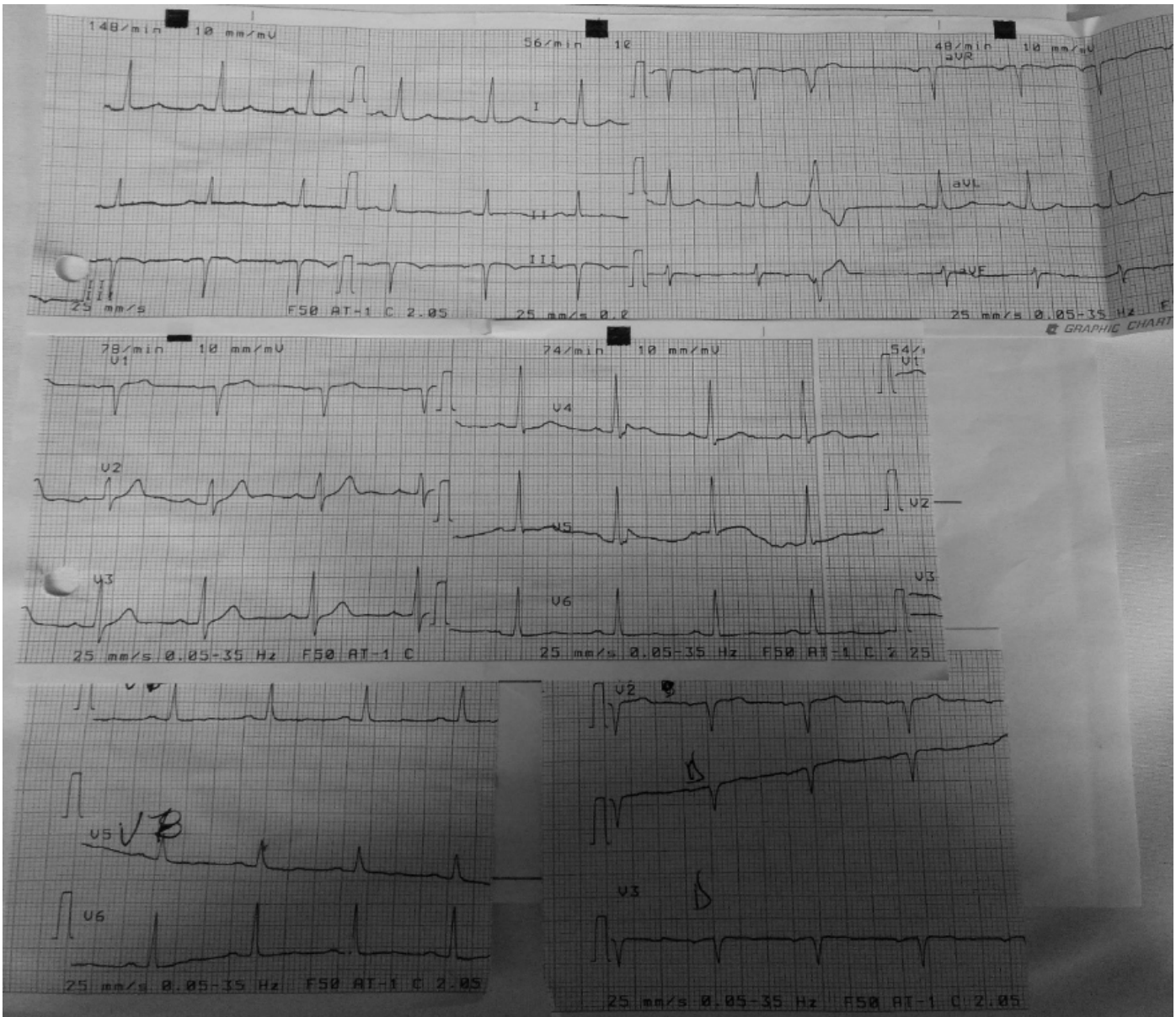

Figure 5. Achieving an ischemic time of 25 minutes, with positive reperfusion criteria (ST reduction of more than $50 \%$, pain disappearance and enzyme elevation, troponin $400 \mathrm{pg} / \mathrm{mL}$ ) 


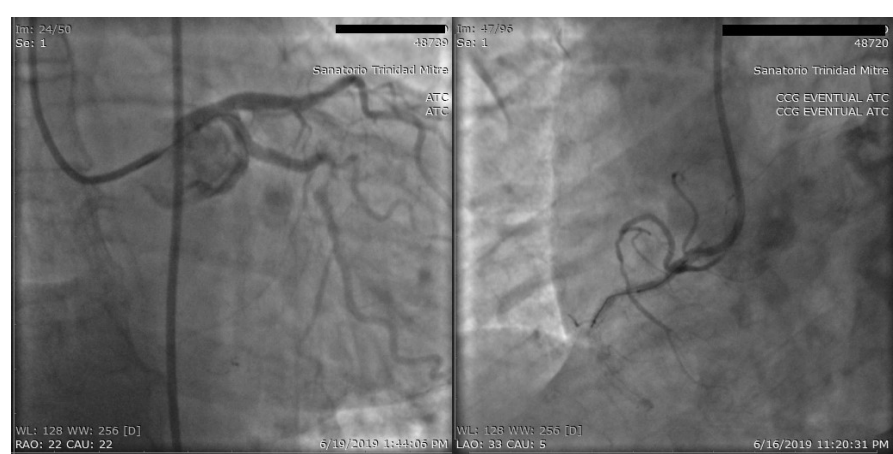

Figure 6. The CCG showed the left main coronary artery without relevant angiographic injuries

\section{Discussion}

There is consensus that the sooner the reperfusion treatment is performed in patients with acute STEMI, the better the short- and median-term results are regarding prognosis and survival [3]. A study compared pre-hospital reperfusion strategy with thrombolytics (ischemic time (IT) 113 minutes) with in-hospital-treated patients (median IT 165 minutes); pre-hospital thrombolysis was associated with reduced 1-year mortality [4]. It is worth mentioning that patients admitted to hemodynamics service, following a fibrinolytic therapy, show a TIMI grade- 3 flow of the culprit artery before PTCA in $80 \%$ of the cases, as shown by the study GRACIA-1 [5]. The NORDISTEMI study revealed no difference in the TIMI flow of the culprit artery between the conservative group and the invasive group (both groups showed a percentage superior to $85 \%$ TIMI 3) [6].

According to the National Survey of ST-Segment Elevation Acute Myocardial Infarction in Argentina (ARGEN-IAM ST), in our country the time-to-reperfusion falls far short of the ideal. The situation is even worse if patients are admitted to centers without intervention capabilities and are then transferred to another center with hemodynamics service (time delay is approximately 2 hours). To sum up, one out of three STEMI patients is transferred to another center for reperfusion therapy. About $40 \%$ of centers lack reperfusion capabilities. Besides, it is important to mention that $65 \%$ of the population included in the registry belongs to the provinces of Buenos Aires, Cordoba and Santa Fe (provinces with more resources available). Probably the situation in the rest of the country is worse. This survey also revealed that patients transferred to another center are less likely to receive reperfusion therapy. Less than half of the patients transferred from another institution were admitted within 3 hours of symptom onset, and, globally, only $16 \%$ of the patients received thrombolytic therapy before being transferred [7].

Taking into account the references and the results obtained in the cases described, we believe there is an important association between both parts, reason why the early strategy of reperfusion with thrombolytics adapts to the reality of our country.

\section{Conclusion}

Considering the scientific evidence available and the time delays to reperfusion with primary percutaneous coronary intervention, we believe it is necessary to strengthen the therapeutic indication of reperfusion with thrombolytics as early as possible, both within the context of a transfer for a pharmaco-invasive reperfusion strategy and in those situations in which it is the only available treatment.

\section{References}

1. Widimsky P, Wijns W, Fajadet J, Belder M, Knot J, et al. (2009) Reperfusion therapy for ST elevation acute myocardial infarction in Europe: description of the current situation in 30 countries. Eur Heart J 31: 943-957. [Crossref]

2. Bonnefoy E, Steg P, Boutitie F, Dubien P, Lapostolle F, et al. (2009) Comparison of primary angioplasty and pre-hospital fibrinolysis in acute myocardial infarction (CAPTIM) trial: a 5-year follow-up. Eur Heart J 30: 1598-1606. [Crossref]

3. Cannon C, Gibson C, Lambrew C, Shoultz D, Levy D, et al. (2000) Relationship of symptom-onset-to-ballon time and door-to-balloon time with mortality in patients undergoing angioplasty for acute myocardial infarction. JAMA 283: 2941-2947. [Crossref]

4. Bjorklund E, Stenestrand U, Lindback J, Svensson L, Wallentin L, et al. (2006) Prehospital thrombolysis delivered by paramedics is associated with reduced time delay and mortality in ambulance-transported real-life patients with ST-elevation myocardial infarction. Eur Heart J 27: 1146-1152. [Crossref]

5. Fernandez-Aviles F, Alonso J, Castro-Beiras A, Vazquez N, Blanco J, et al. (2004) Routine invasive strategy within 24 hours of thrombolysis versus ischaemia-guided conservative approach for acute myocardial infarction with ST-segment elevation (GRACIA-1): a randomised controlled trial. The Lancet 364: 1045-1053. [Crossref]

6. Bøhmer E, Hoffmann P, Abdelnoor M, Arnesen H, Halvorsen S (2009) Efficacy and safety of immediate angioplasty versus ischemia-guided management after thrombolysis in acute myocardial infarction in areas with very long transfer distancesresults of the NORDISTEMI (NORwegian study on DIstrict treatment of ST-elevation myocardial infarction). J Am Coll Cardiol 55: 102-110. [Crossref]

7. Charask A, Castillo Costa Y, Dimperio H, Perna E, Zapata G, et al. (2017) Pacientes con infarto agudo de miocardio con elevación del ST trasladados a centros con hemodinamia. Encuesta Nacional de Infarto Agudo de Miocardio con Elevación del ST en la República Argentina (ARGEN-IAM-ST). Rev Argent Cardiol 85: 90-102.

Copyright: (C2020 Benaim FL. This is an open-access article distributed under the terms of the Creative Commons Attribution License, which permits unrestricted use, distribution, and reproduction in any medium, provided the original author and source are credited. 